Comparison between PUN and Tofts models in the quantification of dynamic contrastenhanced MR imaging

This content has been downloaded from IOPscience. Please scroll down to see the full text. 2012 Phys. Med. Biol. 578443

(http://iopscience.iop.org/0031-9155/57/24/8443)

View the table of contents for this issue, or go to the journal homepage for more

Download details:

IP Address: 130.192.248.36

This content was downloaded on 22/01/2016 at 08:55

Please note that terms and conditions apply. 


\title{
Comparison between PUN and Tofts models in the quantification of dynamic contrast-enhanced MR imaging
}

\author{
S Mazzetti ${ }^{1}$, A S Gliozzi ${ }^{2}$, C Bracco $^{1}$, F Russo $^{1}$, D Regge $^{1}$ and M Stasi ${ }^{1}$ \\ ${ }^{1}$ Institute for Cancer Research and Treatment, Strada Provinciale 142, km 3.95, 10060 Candiolo, \\ Torino, Italy \\ ${ }^{2}$ Department of Applied Science and Technology, Institute of Physics of Condensed Matter and \\ Complex Systems, Politecnico di Torino, C.so Duca degli Abruzzi 24, 10129 Torino, Italy \\ E-mail: simone.mazzetti@ircc.it
}

Received 5 September 2012, in final form 23 October 2012

Published 30 November 2012

Online at stacks.iop.org/PMB/57/8443

\begin{abstract}
Dynamic contrast-enhanced study in magnetic resonance imaging (DCEMRI) is an important tool in oncology to visualize tissues vascularization and to define tumour aggressiveness on the basis of an altered perfusion and permeability. Pharmacokinetic models are generally used to extract hemodynamic parameters, providing a quantitative description of the contrast uptake and wash-out. Empirical functions can also be used to fit experimental data without the need of any assumption about tumour physiology, as in pharmacokinetic models, increasing their diagnostic utility, in particular when automatic diagnosis systems are implemented on the basis of an MRI multiparametric approach. Phenomenological universalities (PUN) represent a novel tool for experimental research and offer a simple and systematic method to represent a set of data independent of the application field. DCE-MRI acquisitions can thus be advantageously evaluated by the extended PUN class, providing a convenient diagnostic tool to analyse functional studies, adding a new set of features for the classification of malignant and benign lesions in computer aided detection systems. In this work the Tofts pharmacokinetic model and the class EU1 generated by the PUN description were compared in the study of DCE-MRI of the prostate, evaluating complexity of model implementation, goodness of fitting results, classification performances and computational cost. The mean $R^{2}$ obtained with the EU1 and Tofts model were equal to 0.96 and 0.90 , respectively, and the classification performances achieved by the EU1 model and the Tofts implementation discriminated malignant from benign tissues with an area under the receiver operating characteristic curve equal to 0.92 and 0.91 , respectively. Furthermore, the EU1 model has a simpler functional form which reduces implementation complexity
\end{abstract}


and computational time, requiring 6 min to complete a patient elaboration process, instead of $8 \mathrm{~min}$ needed for the Tofts model analysis.

(Some figures may appear in colour only in the online journal)

\section{Introduction}

Dynamic contrast-enhanced magnetic resonance imaging (DCE-MRI) is an indispensable tool in oncology for assisting with clinical drug trials and diagnosis (Orton et al 2007). This technique can be used to characterize microvasculature, providing information about the tumour microvessel structure and function (Neeman et al 2001). In fact DCE-MRI is sensitive to a variety of contrast mechanisms, including blood flow, microvessel permeability and diameter, tissue oxygenation and metabolism, which may give useful information about the tumour microenvironment (Padhani 2002).

The ability to extract reliable quantitative information from DCE-MRI data is therefore of great importance and it is typically achieved by applying mathematical pharmacokinetic models (Brix et al 1991, Daldrup et al 1998, Tofts et al 1999) which have a direct relationship with key biological processes of interest such as perfusion, blood volume and capillary permeability (Fan et al 2007). This is a distinct advantage over the semi-quantitative approach where descriptive statistics of the kinetic curve (maximum signal intensity, uptake rate, washout rate, area under the curve) are estimated, but lack direct tissue or vascular information (Schmid et al 2005).

However, pharmacokinetic model implementation typically involves assuming some prior knowledge and requires measuring the arterial input function (AIF). Inaccuracy in the theoretical model and related assumptions lead to potentially large errors in pharmacokinetic parameter estimation. This has clear implications for clinical applications (Orton et al 2007).

Recent studies showed an increasing interest in developing automatic diagnosis systems to detect and characterize prostate cancer on the basis of a multi-parametric MR imaging approach (Vos et al 2012, Niaf et al 2012, Sung et al 2011). The aim was to assist radiologists in making correct diagnosis decisions by providing an objective and reproducible malignancy score for suspicious targets (Niaf et al 2012, Shah et al 2012). In such a framework, in which CAD aims to automatically highlight cancer suspicious regions within a malignancy likelihood map, there is no longer the need of displaying images reporting direct tissue or vascular information (e.g. $K^{\text {trans }}$ or $k_{\text {ep }}$ maps) because lesion candidates are directly accessible to the radiologist through cancer probability maps as the output of the CAD. This way it is possible to study DCE-MRI data by empirical approaches, overcoming problems associated with pharmacokinetic model assumptions and AIF determination. Empirical functions can thus be used to accurately describe DCE-MRI curves without making any assumption about tumour physiology (Fan et al 2007). It was recently demonstrated that the empirical approach based on phenomenological universalities (PUN) is able to reproduce experimental data from a DCE-MRI acquisition (Gliozzi et al 2011).

The objective of this work was to compare the Tofts pharmacokinetic model and the class EU1 (Gliozzi et al 2011), derived from the extended PUN description, when applied to a DCEMRI study of the prostate. Different parameters were exploited in this analysis: complexity of model implementation, fitting results, classification performances and computational time efforts. 


\section{Material and methods}

\section{Study population and MRI protocol}

A series of 28 patients who ranged in age from 47.4 to 75.6 years (mean \pm SD age, $64.7 \pm 7.5$ years), with a prostate specific antigen (PSA) level greater than $4 \mathrm{ng} \mathrm{ml}^{-1}$ and a confirmed prostate tumour by transrectal ultrasonography guided biopsy, were included in this study. MR imaging was performed with a 1.5 T scanner (Signa HDx, GE, Milwaukee, USA) using an endorectal coil. Conventional T1-w and T2-w MRI series were used to locate the prostate and to exhibit its morphologic characteristics. After these preliminary series, a precontrast sagittal 3D spoiled gradient recalled acquisition (SPGR) sequence (TR/TE/NEX/FA: $3.6 \mathrm{~ms} / 1.3 \mathrm{~ms} / 0.5 / 20^{\circ}$, bandwidth: $83.3 \mathrm{kHz}$, slice thickness: $3 \mathrm{~mm}$, acquisition matrix: $256 \times 192$, reconstruction matrix: $512 \times 512$, field of view: $20 \times 20 \mathrm{~cm}$ ) was used as the baseline image for DCE-MRI acquisitions. After the precontrast sequence, patients were given $0.1 \mathrm{mmol} \mathrm{kg}^{-1}$ gadobutrol (Gadovist, Bayer Schering, Berlin, Germany) intravenously through a peripheral line at $0.7 \mathrm{ml} \mathrm{s}^{-1}$, using a power injector (Medrad Spectris, Maastricht, The Netherlands), followed by an infusion of $20 \mathrm{cc}$ normal saline at the same velocity. In the contrast sequence, a multiphase sagittal 3D SPGR was applied with parameters identical to those used during the precontrast sequence. For each patient a total of 25 phases were acquired sequentially, each lasting $13 \mathrm{~s}$, for a total acquisition time of approximately 6 min.

\section{Reference standard}

All patients underwent radical prostatectomy treatment for prostate cancer within 3 months from the MR imaging session. Each prostate was divided into axial sections of the same thickness and orientation of the MR images. Foci of cancer were contoured on each slice by the pathologist who evaluated the presence of cancer. The Gleason scores of the detected cancers in this study population were 6 (8 patients), 7 (19 patients) and 8 (1 patient). Images collected during DCE-MRI exams and digitalized histopathology sections were stored in a workstation for data analysis. Regions of interest (ROIs) were manually drawn for each patient on MR images by an experienced radiologist on the basis of foci marked by the pathologist on the prostate specimens. When the tumour extended on more slices, multiple ROIs were considered, covering the whole lesion. Benign prostate regions were also selected for each patient to sample benign tissues behaviour on DCE-MRI curves.

\section{Data pre-processing and noise filtering}

Signal intensity over the acquisition time course was considered for each pixel belonging the ROIs drawn by the radiologist. The dynamic curve dataset was normalized to the corresponding pixel precontrast value.

A noise filtering operation was performed on resulting curves with a discrete stationary wavelet transform: a four-level signal decomposition using the Daubechies filter (Coifman and Donoho 1995) was applied with hard thresholding on wavelet coefficients and a leveldependent threshold selection method (Zhong and Cherkassky 2000). Finally the inverse wavelet transform was performed to obtain corresponding denoised signals. The filtering operation was introduced in the post-processing steps to reduce noise contribution and to get more reliable results during the fitting procedure and parameter extraction. 
Signal-to-noise ratio (SNR) was computed according to equation (1):

$$
\mathrm{SNR}=10 \log \left(\frac{P_{\text {signal }}}{P_{\text {noise }}}\right)
$$

where $P_{\text {signal }}$ is the power of the signal without the filtering step and $P_{\text {noise }}$ the power of the noise computed as

$$
P_{\text {signal }}=\sum_{i} s_{i}^{2} ; \quad P_{\text {noise }}=\sum_{i} r_{i}^{2}
$$

in which $s_{i}$ is the $i$ th original signal point, $r_{i}$ is the difference between the $i$ th original and filtered points. SNR estimation was useful to discard noisy curves before fitting procedure; in fact only curves satisfying the condition SNR $>10 \mathrm{~dB}$ were included in statistical analysis.

\section{Tofts pharmacokinetic model application}

The first model implemented in this study to describe DCE-MRI curves was the Tofts pharmacokinetic model. It is based on some basic assumptions related to concepts in tracer kinetics and MR theory (Patlak et al 1983, Tofts 1997). The principal assumption is that human body tissues may be represented by one or more 'compartments', into and out of which the contrast agent (CA) dynamically flows. A compartment may be defined as a bounded space that the CA can occupy and whose volume remains constant on the time scale of the DCE-MRI experiment (Yankeelov and Gore 2009). A second assumption is that each compartment is assumed to be 'well-mixed' in the sense that the CA entering the compartment is immediately distributed uniformly throughout it (Yankeelov and Gore 2009). In this work two compartments were considered: the first was the extracellular-extravascular space (EES) and the other the intravascular space. After intravenous injection, the CA is rapidly distributed throughout the vasculature volume and extravasated into the EES in a reversible process characterized by a distribution rate constant $\left(K^{\text {trans }}\right)$ and a redistribution rate constant $\left(k_{\mathrm{ep}}\right)$. Since there is evidence that no metabolic trapping of CA occurs within the body (Radjenovic et al 2008), it is completely eliminated in an unchanged form by renal excretion. Pharmacokinetic parameters $K^{\text {trans }}$ and $k_{\text {ep }}$, therefore, reflect the process of CA transfer across the capillary walls and are thus related to their permeability. The functional form of the implemented Tofts model is reported in equation (3) (Tofts 1997, Cheng 2009):

$$
C_{t}(t)=v_{p} C_{p}(t)+K^{\mathrm{trans}} \int_{0}^{t} C_{p}(\tau) \mathrm{e}^{-k_{\mathrm{ep}}(t-\tau)} \mathrm{d} \tau
$$

where $C_{t}$ is the tracer concentration in tissue, $v_{p}$ the blood plasma fractional volume per unit volume of tissue and $C_{p}$ the tracer concentration in blood plasma (AIF). The first term denotes the vascular tracer, and the second one the tracer in EES as a result of an exchange between the two compartments.

A technically demanding portion of the data acquisition process in DCE-MRI is acquiring an estimate of CA concentration in blood plasma as a function of time, $C_{p}(t)$. A method to obtain the AIF is from the DCE-MRI dataset itself, simultaneously measuring signal intensity changes (due to the CA passage) in both blood and tissues. A second method assumes that the AIF is similar for all subjects: it is first measured via blood samples in a cohort of subjects and the resulting average AIF is then assumed to be valid for subsequent studies (Yankeelov and Gore 2009). In our study the AIF was obtained using a previously published population AIF (Parker et al 2006).

According to equation (3), there are three unknowns $\left(v_{p}, K^{\text {trans }}\right.$ and $\left.k_{\mathrm{ep}}\right)$, while $C_{t}$ and $C_{p}$ are inputs of the model. Signal intensities of both malignant and benign ROIs $\left(C_{t}\right)$ were considered 
pixelwise. Conversion of signals to concentrations was performed using the methodology described by Medved et al (2004) and summarized in equation (4):

$$
C_{t}(t) \approx \frac{1}{r_{1}} \frac{S(t)-S(0)}{T_{1 \mathrm{ref}} S_{\mathrm{ref}}(0)},
$$

where $r_{1}$ denotes the relaxivity of the CA, $T_{1 \text { ref }}$ the value of $T_{1}$ in the reference tissue, and $S(0)$ and $S_{\text {ref }}(0)$ are the initial (precontrast) signals in the tissue of interest and the reference one, respectively. In our study $r_{1}$ was set to $5.2 \mathrm{mM}^{-1} \mathrm{~s}^{-1}$ (Rohrer et al 2005) and $T_{1 \mathrm{ref}}=1.020 \mathrm{~s}$ for urine content in bladder as reference (Rosenkrantz et al 2011). For each curve, the Tofts pharmacokinetic model was fitted to concentration data points by nonlinear regression using the Levenberg-Marquardt optimization method with initial parameters estimate by the grid search algorithm.

\section{Application of the PUN formalism to DCE-MRI}

The second model implemented to study DCE-MRI curves was based on the PUN approach proposed by Delsanto and collaborators (Castorina et al 2006, Delsanto 2007). Such a method is a convenient empirical tool to analytically represent the experimental data of any given dataset, independently of the application field. This procedure found applications in a wide range of applied science: auxology (Delsanto et al 2008), tumour growth (Gliozzi et al 2010, 2009), nonlinear elasticity (Delsanto et al 2009) and others (Pugno et al 2008, Barberis et al 2010).

Recently, the formalism described in PUN theory has been applied for the evaluation of DCE-MRI studies (Gliozzi et al 2011) in which it was demonstrated that the class EU1 is capable to reproduce all curve types one can obtain in a DCE-MRI session (van Rijswijk et al 2004). The application of a PUN description to experimental points is then a valid alternative to pharmacokinetic models, since it is based on an empirical approach and without the need of any assumptions about tumour physiology or AIF determination (Gliozzi et al 2011).

The EU1 class is characterized by three fitting parameters, $a_{0}, \beta$ and $r$, and the model implemented is reported in equation (5):

$$
y_{\mathrm{EU} 1}(t)=\exp \left[r t+\frac{1}{\beta}\left(a_{0}-r\right)(\exp \beta t)-1\right],
$$

in which $a_{0}$ controls the steepness of the curve at $t=0$ and together with $\beta$ it primarily affects the growth rate of the curve in its first part; $\beta$ is in inverse proportion to the time the system takes to reach the knee of the curve and $r$ determines the behaviour of the second part of the curve: its absolute value is linked to the rapidity of the change and for $r>0$ one can observe a further enhancement of the signal intensity, while for $r<0$ there is a wash-out phase (Gliozzi et al 2011).

The same fitting procedure as for the Tofts model was applied for the EU1 model on concentration curves, and parameters were saved for each patient.

\section{Statistical analysis}

Goodness of fit was tested pixel by pixel employing the standard $R^{2}$ criterion for both Tofts and EU1 models, measuring mean $R^{2}$ values $(\mu)$ and standard deviations $(\sigma)$.

After the fitting procedure, Tofts features $\left(v_{p}, K^{\text {trans }}, k_{\text {ep }}\right)$ and EU1 ones $\left(a_{0}, \beta, r\right)$ were associated with each pixel, classified belonging either to a malignant or a benign ROI. The area under the receiver operating characteristic (ROC) curve was evaluated for each single parameter to assess the ability of each model to correctly classify a pixel as belonging to a 
Table 1. Means and standard deviations for the parameter $K^{\text {trans }}$ (Tofts model) and $a_{0}$ (EU1 model) computed on both tumoural and benign tissues; $p$-values are also reported for each parameter.

\begin{tabular}{lccc}
\hline Parameter & Tumoural tissue & Benign tissue & $p$-value \\
\hline$K^{\text {trans }}$ & $(0.34 \pm 0.12) \mathrm{min}^{-1}$ & $(0.19 \pm 0.06) \mathrm{min}^{-1}$ & $<0.001$ \\
$a_{0}$ & $(18.64 \pm 9.84) \mathrm{min}^{-1}$ & $(4.58 \pm 4.41) \mathrm{min}^{-1}$ & $<0.001$ \\
\hline
\end{tabular}

malignant tumour. According to the ROC analysis, the parameter displaying the highest area under the ROC curve was selected among Tofts features and EU1 ones. Means and standard deviations for these two parameters were calculated, distinguishing between the tumoural and the benign datasets. The Kolmogorov-Smirnov normality test was carried out on the two features with the highest area under the ROC curve. Both of them did not reject the hypothesis of normality, with a 5\% significance level. The presence of a difference between benign and malignant groups of pixels was then assessed by the Student's $t$-test, considering $p<0.05$ as a statistically significant result.

A logistic regression was constructed using two risk estimation models to compare their performance in predicting prostate cancer. Both models used the presence or absence of cancer as the dependent variable and the pair $\left(K^{\text {trans }}, k_{\mathrm{ep}}\right)$ or $\left(a_{0}, r\right)$ as the independent variable. To quantify the quality of the predictors based on each model, the ROC curves were generated and the areas under the ROC curves were computed.

The relationship between the parameters of the pairs $\left(K^{\text {trans }}, a_{0}\right)$ and $\left(k_{\mathrm{ep}}, r\right)$ was evaluated with Pearson's correlation coefficient. Finally the computational time was evaluated to determine the cost needed for a complete exam analysis applying the Tofts as well as EU1 model.

\section{Results}

A total number of 99140 curves were analysed with a resulting SNR equal to $(19.15 \pm 5.49) \mathrm{dB}$. Approximately $92 \%$ of the dataset had an SNR $>10 \mathrm{~dB}$ and corresponding $R^{2}$ of such dataset was $0.902 \pm 0.090$ for the Tofts model and $0.957 \pm 0.073$ for the EU1 description. The best performing parameter among the Tofts triplet was $K^{\text {trans }}$ with an area under the ROC curve equal to $0.873 \pm 0.068$; among EU1 fitting parameters, $a_{0}$ displayed the greatest ROC area equal to $0.897 \pm 0.061$. The mean value and standard deviation for the $K^{\text {trans }}$ parameter (Tofts model) and $a_{0}$ (EU1 model), distinguishing between tumoural and benign tissues, are reported in table 1 . A statistically significant difference in parameter values between benign and malignant regions was found $(p<0.05)$.

The distributions of the pair $\left(K^{\text {trans }}, k_{\text {ep }}\right)$ for the Tofts and $\left(a_{0}, r\right)$ for the EU1 model are shown in the scatter plots reported in figure 1 . The results of the logistic regression, implementing a multi-parametric analysis, distinguished the data as benign or malignant with an area under the ROC curve equal to $0.913 \pm 0.093$ for the Tofts and $0.921 \pm 0.085$ for the EU1 model. Correlation coefficients between the features $\left(K^{\text {trans }}, a_{0}\right)$ and $\left(k_{\text {ep }}, r\right)$ were equal to 0.83 and -0.90 , respectively.

The mean time for the Tofts curve fitting was $(0.0296 \pm 0.0050) \mathrm{s}$ per pixel. The EU1 model required $(0.0231 \pm 0.0049) \mathrm{s}$ per pixel, approximately $20 \%$ less time than Tofts implementation. The time needed for a complete patient elaboration was approximately 8 min for the Tofts model and 6 min using the EU1 model, considering a mean number of 10000 pixels per patient and including the time required to give the initial parameters' estimate. 


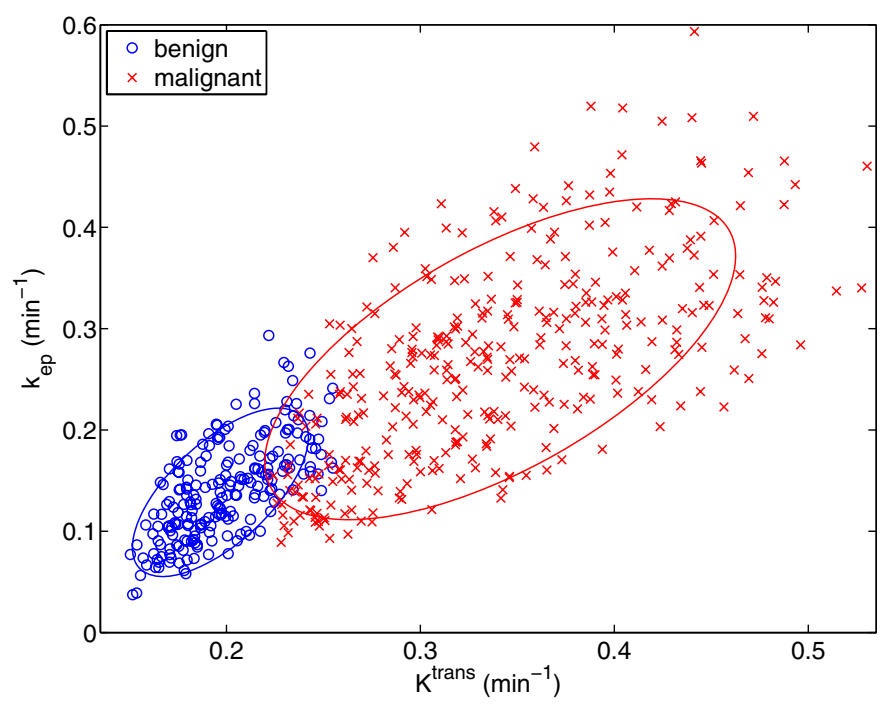

(a)

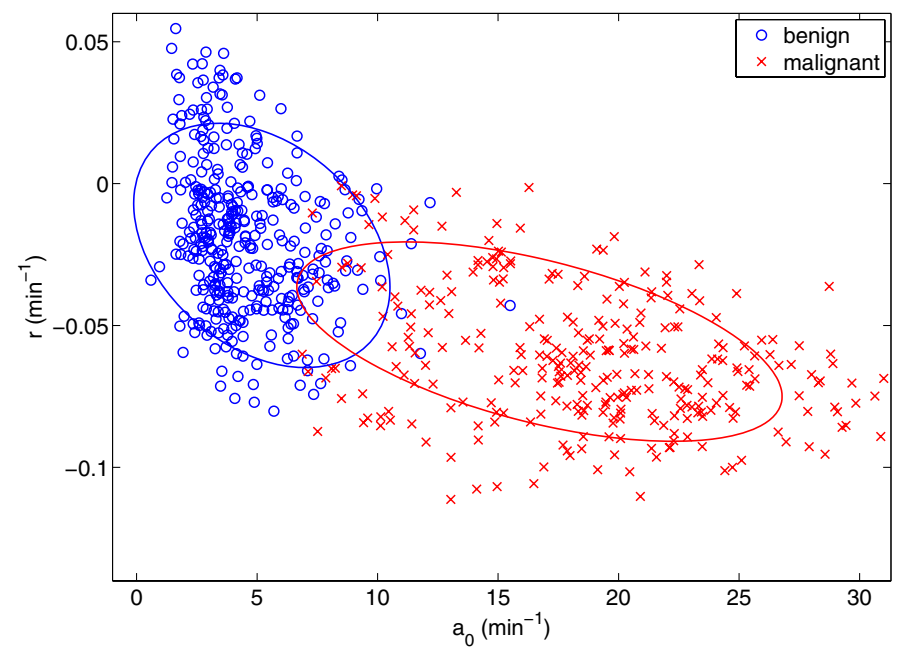

(b)

Figure 1. Features distributions in the parametric planes $\left(K^{\text {trans }}, k_{\mathrm{ep}}\right)$ for $(\mathrm{a})$ the Tofts pharmacokinetic model and $\left(a_{0}, r\right)$ for $(b)$ the EU1 empirical model. Both benign (circles) and malignant (crosses) markers are shown. The ellipses summarize the two clusters by fitting a bivariate normal distribution and displaying the outline at two times standard deviation radius. The clustering of features found in the Tofts parametric plane is also well defined in the EU1 description of dynamic curves.

\section{Discussion}

The differentiation between non-malignant and malignant tissues is a recurring clinical challenge in diagnostic radiology (Rudisch et al 2005). Advances in MR image acquisition have led to an increasing use of parametric or calculated images, designed to display physiological features of tissues rather than simply anatomical structures (Jackson 2004). One common type of dynamic imaging data is the DCE-MRI technique which is increasingly applied for 
the non-invasive characterization of tumour microvasculature, improving detection, staging, treatment planning and early evaluation of response to anti-cancer treatments (Aerts et al 2011, Fan et al 2007, Padhani 2002, Yankeelov et al 2005).

Methods for quantifying change in enhancement can be broadly classified into two categories: pharmacokinetic models and empirical parametric models. The former attempt to quantify the observed CA kinetics in terms of physiologically meaningful parameters whilst the latter seek to reproduce the shape of the CA time course in terms of simple quantitative parameters (Jackson et al 2005). Each enhancement kinetic curve is characterized by three distinct features: the wash-in phase, the maximum enhancement and the wash-out rate. Wash-in is associated with high perfusion and/or high permeability; maximum enhancement is related to the total uptake in the interstitial space; and the wash-out rate is associated with the CA clearance and may be related in part to vascular permeability ( $\mathrm{Su}$ et al 2003). Pharmacokinetic models (Tofts et al 1999, Jackson et al 2004) describe the wash-in and wash-out phases of a CA from the microvasculature into the surrounding interstitial space in both tumour and normal tissue (Aerts et al 2011). Interest in DCE-MRI was stimulated by early observations that both high increases in enhancement and fast rates of signal enhancement correlate with well-vascularized, viable tumour regions, compared to more moderate rates and extents of signal increase in many normal tissues and benign lesions (Taylor and Reddick 2000, d'Arcy et al 2006, Yankeelov et al 2005). Pharmacokinetic parameters are quantitative insofar as their potential to measure true physiology, such as blood flow and endothelial permeability. However, this potential is difficult to realize in practice due to several challenges, including parameter coupling, measuring the AIF, water exchange and model fit instability (Cheng 2009). Many clinical investigations usually do not analyse DCE-MRI using a pharmacokinetic model, but rather have extracted a variety of empirical parameters as measures (Taylor and Reddick 2000, Mehnert et al 2010). Another attempt to describe the signal intensity versus time curves is using an empirical mathematical model (EMM) to accurately fit contrast uptake and washout behaviours (Fan et al 2007, Gal et al 2007). It was demonstrated that parameters derived from EMM distinguish between metastatic and non-metastatic rodent prostate tumours more reliably than the two-compartment model approach (Fan et al 2004).

In this work, we compared the Tofts pharmacokinetic model with the EU1 empirical function derived from the PUN description (Gliozzi et al 2011). We chose to use two functions characterized by the same number of fitting parameters and implemented a pixel-by-pixel analysis. The first one was the Tofts two-compartment kinetic model, used to describe the transport of the CA between vascular and interstitial spaces as a function of time. The three fitting parameters were the transfer constant $\left(K^{\text {trans }}\right.$, often called the wash-in rate and related to the forward rate of the contrast medium), the diffusion back into the vasculature $\left(k_{\mathrm{ep}}\right.$, also called the wash-out rate) from where the CA is excreted by the kidneys and the plasma volume fraction $\left(v_{p}\right)$ (Padhani and Khan 2010).

However, tumours are extremely heterogeneous on a microscopic level and pharmacokinetic models are frequently incompatible with experimental data (Fan et al 2004). Application of an empirical model has the advantage of not requiring any assumptions about tumour physiology or a functional form for the AIF (Yankeelov and Gore 2009, Orton et al 2007), albeit they exhibit flexibility to accurately describe dynamic curves for long periods of time and in different types of tissues. The extended universalities class was able to reproduce contrast uptake and wash-out phases giving high $R^{2}$ values. The class EU1 is then a convenient analytical curve to represent our dataset and it provides a model to interpret a DCE-MRI experiment.

The implemented empirical model has the same number of fitting parameters as the pharmacokinetic model, i.e. three, and one could attempt to correlate them with Tofts features. 
The wash-in phase is responsible for the very first part of the dynamic curve in which $K^{\text {trans }}$ is the dominant contribution. In a similar way $a_{0}$ affects the steepness of the curve at $t=0$, so one can consider that the greater $K^{\text {trans }}$, the larger $a_{0}$ would be. In fact, figure 1 shows that malignant pixels are characterized by large $a_{0}$ values, indicating that the enhancement ratio is a basic clinical tool for assessing tumour vasculature. Analogous similarity can be found between the wash-out phase and parameter $r$, responsible for the behaviour of the second part of the curve, after the enhancement peak, where the CA diffuses back into the vasculature.

The separation power between tumoural and benign pixels was evaluated for both the Tofts and EU1 models computing the area under the ROC curve, which was equal to 0.91 and 0.92 , respectively. These results demonstrated the validity of an empirical curve description over a pharmacokinetic analysis in a DCE-MRI experiment. Furthermore, the power of the proposed approach is the ability to describe the whole process arising from vascularization of a suspected region using a few parameters (whose interpretation is quite simple) and without making any assumption on tissue physiology. Therefore, the EU1 application to dynamic curve analysis can be very useful in the diagnosis of a pathology, in particular when the AIF is not available in the MR field of view.

The pixel-by-pixel analysis was chosen to include the possibility of evaluating heterogeneities within a lesion in further studies. In fact, although this is a time-consuming process, it may offer additional information to improve tumour vascular assessment. When a quantitative evaluation is required, another important issue to account for is whether a better temporal resolution can improve the determination of vascular parameters and strengthen the correlation with vascular density. However, in clinical practice, fast sampling conditions give rise to suboptimal spatial resolution, spatial coverage and signal-to-noise characteristics and hamper the detection of contrast enhancement and the visualization of the underlying anatomic details (Aerts et al 2011). Therefore, a better spatial resolution is generally more important than a better temporal resolution, especially when smaller lesions detection is a demanding question ( $\mathrm{Su}$ et al 2003). On the other hand, it was demonstrated that as the temporal resolution decreases, $K^{\text {trans }}$ is progressively underestimated and the fractional EES is progressively overestimated (Heisen et al 2010). Hence a further development of this study could be the implementation of high-temporal-resolution DCE-MRI to investigate if a shorter temporal resolution would result in a better separation between malignant tissues and benign ones, applying both pharmacokinetic and empirical analyses.

\section{Conclusion}

The application of an empirical model to DCE-MRI data is a useful alternative to pharmacokinetic modelling. Empirical models have no need of an AIF, which are known to affect the accuracy of the fitted parameters in modelling and add to the computational complexity. The EU1 model is simple to implement and discriminates malignant from benign tissues as well as an established pharmacokinetic model.

\section{References}

Aerts H J, Jaspers K and Backes W H 2011 The precision of pharmacokinetic parameters in dynamic contrast-enhanced magnetic resonance imaging: the effect of sampling frequency and duration Phys. Med. Biol. 56 5665-78

Barberis L, Condat C A, Gliozzi A S and Delsanto P P 2010 Concurrent growth of phenotypic features: a phenomenological universalities approach J. Theor. Biol. 264 123-9

Brix G, Semmler W, Port R, Schad L R, Layer G and Lorenz W J 1991 Pharmacokinetic parameters in CNS Gd-DTPA enhanced MR Imaging J. Comput. Assist. Tomogr. 15 621-8 
Castorina P, Delsanto P P and Guiot C 2006 Classification scheme for phenomenological universalities in growth problems in physics and other sciences Phys. Rev. Lett. 96188701

Cheng H L 2009 Improved correlation to quantitative DCE-MRI pharmacokinetic parameters using a modified initial area under the uptake curve (mIAUC) approach J. Magn. Reson. Imaging 30 864-72

Coifman R R and Donoho D L 1995 Translation-invariant noising Wavelets and Statistics (Lecture Notes in Statistics vol 103) ed A Antoniadis and G Oppenheim (New York: Springer) pp 125-50

d'Arcy J A, Collins D J, Padhani A R, Walker-Samuel S, Suckling J and Leach M O 2006 Informatics in radiology (infoRAD): magnetic resonance imaging workbench: analysis and visualization of dynamic contrast-enhanced MR imaging data Radiographics 26 621-32

Daldrup H E, Shames D M, Husseini W, Wendland M F, Okuhata Y and Brasch R C 1998 Quantification of the extraction fraction for gadopentetate across breast cancer capillaries Magn. Reson. Med. 40 537-43

Delsanto P P (ed) 2007 Universality of Nonclassical Nonlinearity with Applications to NDE and Ultrasonics (New York: Springer)

Delsanto P P, Gliozzi A S and Guiot C 2008 Scaling, growth and cyclicity in biology: a new computational approach Theor. Biol. Med. Modelling 55

Delsanto P P, Gliozzi A S, Bruno C L E, Pugno N and Carpinteri A 2009 Scaling laws and fractality in the framework of a phenomenological approach Chaos Solitons Fractals 41 2782-6

Fan X, Medved M, River J N, Zamora M, Corot C, Robert P, Bourrinet P, Lipton M, Culp R M and Karczmar G S 2004 New model for analysis of dynamic contrast-enhanced MRI data distinguishes metastatic from nonmetastatic transplanted rodent prostate tumors Magn. Reson. Med. 51 487-94

Fan X, Medved M, Karczmar G S, Yang C, Foxley S, Arkani S, Recant W, Zamora M A, Abe H and Newstead G M 2007 Diagnosis of suspicious breast lesions using an empirical mathematical model for dynamic contrastenhanced MRI Magn. Reson. Imaging 25 593-603

Gal Y, Mehnert A, Bradley A, McMahon K and Crozier S 2007 An evaluation of four parametric models of contrast enhancement for dynamic magnetic resonance imaging of the breast Conf. Proc. IEEE Engineering Medicine Biology Society (Lyon, France) pp 71-4

Gliozzi A S, Guiot C and Delsanto P P 2009 A new computational tool for the phenomenological analysis of multipassage tumor growth curves PLoS One 4 e5358

Gliozzi A S, Guiot C, Chignola R and Delsanto P P 2010 Oscillations in growth of multicellular tumour spheroids: a revisited quantitative analysis Cell Prolif. 43 344-53

Gliozzi A S, Mazzetti S, Delsanto P P, Regge D and Stasi M 2011 Phenomenological universalities: a novel tool for the analysis of dynamic contrast enhancement in magnetic resonance imaging Phys. Med. Biol. $56573-86$

Heisen M, Fan X, Buurman J, van Riel N A, Karczmar G S and ter Haar Romeny B M 2010 The influence of temporal resolution in determining pharmacokinetic parameters from DCE-MRI data Magn. Reson. Med. 63 811-6

Jackson A 2004 Analysis of dynamic contrast enhanced MRI Br. J. Radiol. 77 S154-66

Jackson A, Buckley D L and Parker G J M (ed) 2005 Dynamic Contrast-Enhanced Magnetic Resonance Imaging in Oncology (Berlin: Springer)

Medved M, Karczmar G, Yang C, Dignam J, Gajewski T F, Kindler H, Vokes E, MacEneany P, Mitchell M T and Stadler W M 2004 Semiquantitative analysis of dynamic contrast enhanced MRI in cancer patients: variability and changes in tumor tissue over time J. Magn. Reson. Imaging 20 122-8

Mehnert A, Wildermoth M, Crozier S, Bengtsson E and Kennedy D 2010 Two non-linear parametric models of contrast enhancement for DCE-MRI of the breast amenable to fitting using linear least squares DICTA: Int. Conf. on Digital Image Computing: Techniques and Applications, (Sydney, Australia, 1-3 Dec. 2010) pp 611-6

Neeman M, Provenzale J M and Dewhirst M W 2001 Magnetic resonance imaging applications in the evaluation of tumor angiogenesis Semin. Radiat. Oncol. 11 70-82

Niaf E, Rouvire O, Mge-Lechevallier F, Bratan F and Lartizien C 2012 Computer-aided diagnosis of prostate cancer in the peripheral zone using multiparametric MRI Phys. Med. Biol. 57 3833-51

Orton M R, Collins D J, Walker-Samuel S, d'Arcy J A, Hawkes D J, Atkinson D and Leach M O 2007 Bayesian estimation of pharmacokinetic parameters for DCE-MRI with a robust treatment of enhancement onset time Phys. Med. Biol. 52 2393-408

Padhani A R 2002 Dynamic contrast-enhanced MRI in clinical oncology: current status and future directions J. Magn. Reson. Imaging 16 407-22

Padhani A R and Khan A A 2010 Diffusion-weighted (DW) and dynamic contrast-enhanced (DCE) magnetic resonance imaging (MRI) for monitoring anticancer therapy Targeted Oncol. 5 39-52 
Parker G J, Roberts C, Macdonald A, Buonaccorsi G A, Cheung S, Buckley D L, Jackson A, Watson Y, Davies K and Jayson G C 2006 Experimentally-derived functional form for a population-averaged high-temporalresolution arterial input function for dynamic contrast-enhanced MRI Magn. Reson. Med. 56 993-1000

Patlak C S, Blasberg R G and Fenstermacher J D 1983 Graphical evaluation of blood-to-brain transfer constants from multiple-time uptake data J. Cereb. Blood Flow Metab. 3 1-7

Pugno N, Bosia F, Gliozzi A S, Delsanto P P and Carpinteri A 2008 Phenomenological approach to mechanical damage growth analysis Phys. Rev. E 78046103

Radjenovic A, Dall B J, Ridgway J P and Smith M A 2008 Measurement of pharmacokinetic parameters in histologically graded invasive breast tumours using dynamic contrast-enhanced MRI Br. J. Radiol. 81 120-8

Rohrer M, Bauer H, Mintorovitch J, Requardt M and Weinmann H J 2005 Comparison of magnetic properties of MRI contrast media solutions at different magnetic field strengths Invest. Radiol. 40 715-24

Rosenkrantz A B, Niver B E, Kopec M, Berkman D S, Lepor H, Babb J S and Hecht E M 2011 T1 hyperintensity of bladder urine at prostate MRI: frequency and comparison with urinalysis findings Clin. Imaging 35 203-7

Rudisch A, Kremser C, Judmaier W, Zunterer H and DeVries A F 2005 Dynamic contrast-enhanced magnetic resonance imaging: a non-invasive method to evaluate significant differences between malignant and normal tissue Eur. J. Radiol. 53 514-9

Schmid V J, Whitcher B J, Yang G Z, Taylor N J and Padhani A R 2005 Statistical analysis of pharmacokinetic models in dynamic contrast-enhanced magnetic resonance imaging Lect. Notes Comput. Sci. 3750 886-93

Shah V, Turkbey B, Mani H, Pang Y, Pohida T, Merino M J, Pinto P A, Choyke P L and Bernardo M 2012 Decision support system for localizing prostate cancer based on multiparametric magnetic resonance imaging Med. Phys. 39 4093-103

Su M Y et al 2003 Correlation of dynamic contrast enhancement MRI parameters with microvessel density and VEGF for assessment of angiogenesis in breast cancer J. Magn. Reson. Imaging 18 467-77

Sung Y S, Kwon H J, Park B W, Cho G, Lee C K, Cho K S and Kim J K 2011 Prostate cancer detection on dynamic contrast-enhanced MRI: computer-aided diagnosis versus single perfusion parameter maps AJR Am. J. Roentgenol. 197 1122-9

Taylor J S and Reddick W E 2000 Evolution from empirical dynamic contrast-enhanced magnetic resonance imaging to pharmacokinetic MRI Adv. Drug Deliv. Rev. 41 91-110

Tofts P S 1997 Modeling tracer kinetics in dynamic Gd-DTPA MR imaging J. Magn. Reson. Imaging 7 91-101

Tofts P S et al 1999 Estimating kinetic parameters from dynamic contrast-enhanced T1-weighted MRI of a diffusible tracer: standardized quantities and symbols J. Magn. Reson. Imaging 10 223-32

van Rijswijk C S, Geirnaerdt M J, Hogendoorn P C, Taminiau A H, van Coevorden F, Zwinderman A H, Pope T L and Bloem J L 2004 Soft-tissue tumors: value of static and dynamic gadopentetate dimeglumine-enhanced MR imaging in prediction of malignancy Radiology 233 493-502

Vos P C, Barentsz J O, Karssemeijer N and Huisman HJ 2012 Automatic computer-aided detection of prostate cancer based on multiparametric magnetic resonance image analysis Phys. Med. Biol. 57 1527-42

Yankeelov T E, Luci J J, Lepage M, Li R, Debusk L, Lin P C, Price R R and Gore J C 2005 Quantitative pharmacokinetic analysis of DCE-MRI data without an arterial input function: a reference region model Magn. Reson. Imaging 23 519-29

Yankeelov T E and Gore J C 2009 Dynamic contrast enhanced magnetic resonance imaging in oncology: theory, data acquisition, analysis, and examples Curr. Med. Imaging Rev. 3 91-107

Zhong S and Cherkassky V 2000 Image denoising using wavelet thresholding and model selection Int. Conf. on Image Processing, (Vancouver, Canada, 2000) vol 3 pp 262-5 